\title{
ENTIRE $s$-HARMONIC FUNCTIONS ARE AFFINE
}

\author{
MOUHAMED MOUSTAPHA FALL
}

(Communicated by Mark M. Meerschaert)

ABStRACT. In this paper, we prove that solutions to the equation $(-\Delta)^{s} u=0$ in $\mathbb{R}^{N}$, for $s \in(0,1)$, are affine. This allows us to prove the uniqueness of the Riesz potential $|x|^{2 s-N}$ in Lebesgue spaces.

\section{INTRODUCTION}

The classical Liouville theorem for harmonic functions states that a bounded harmonic function in $\mathbb{R}^{N}$ is constant; see for instance the particularly short proof by E. Nelson in [13. The stronger version of it states that a nonnegative harmonic function on $\mathbb{R}^{N}$ is constant. In the case of the fractional Laplacian $-(-\Delta)^{s}$, for $s \in(0,1)$, (see Section 2) the strong form of the Liouville theorem holds as well and was proved by K. Bogdan et al. in [5]. Applications of Liouville theorems for nonlocal operators in the study of nonlocal elliptic systems of equations can be found in $2,9,14,15]$.

The aim of this paper is to classify all $s$-harmonic functions in $\mathbb{R}^{N}$, thereby obtaining the Liouville theorem for the fractional Laplacian as a particular case.

Theorem 1.1. Every s-harmonic function in $\mathbb{R}^{N}$ is affine, and constant if $s \in$ $(0,1 / 2]$.

The proof of this theorem is mainly based on a Cauchy-type estimate for the derivatives of an $s$-harmonic function. More precisely, given $s \in(0,1), \gamma \in \mathbb{N}^{N}$ and a function $u$ which is $s$-harmonic in the ball $B(0, R)$, we have the estimate

$$
\left|D^{\gamma} u(0)\right| \leq C R^{2 s-|\gamma|} \int_{|y| \geq R / 4}|u(y)||y|^{-N-2 s} d y
$$

for some positive constant $C$ depending only on $N, \gamma$ and $s$; see Section 3 This estimate is obtained from the Poisson kernel representation formula for $s$-harmonic functions. We refer to Section 2 for more details.

Received by the editors July 24, 2014 and, in revised form, April 9, 2015 and August 1, 2015. 2010 Mathematics Subject Classification. Primary 35R11, 42B37.

Key words and phrases. Fractional Laplacian, Liouville theorem, uniqueness, Riesz kernel, entire $\alpha$-harmonic, Cauchy estimates.

This work was supported by the Alexander von Humboldt foundation and the author would like to thank Tobias Weth and Krzysztof Bogdan for useful discussions. This work was completed while the author was visiting the Goethe-Universität Frankfurt am Main and the Technische Universität Chemnitz. The author is also very grateful to the referee for the detailed comments. The variety of substantial suggestions helped the author to improve the earlier versions of this manuscript. 
In the following, we denote by $\mathcal{D}^{\prime}\left(\mathbb{R}^{N}\right)$ the dual of $C_{c}^{\infty}\left(\mathbb{R}^{N}\right)$ endowed with the usual topology. An iteration argument based on Theorem 1.1 allows us to state the following result.

Theorem 1.2. Assume that $s \in(0,1)$ and let $u$ be a solution to the equation

$$
(-\Delta)^{s} u=P \quad \text { in } \mathcal{D}^{\prime}\left(\mathbb{R}^{N}\right),
$$

where $P$ is a polynomial. Then $u$ is affine and $P=0$.

Another consequence of the main theorem which is of independent interest is the following result.

Corollary 1.3. Let $p \in[1, \infty)$ and $u \in L^{p}\left(\mathbb{R}^{N}\right)$ be such that

$$
(-\Delta)^{s} u=0 \quad \text { in } \mathcal{D}^{\prime}\left(\mathbb{R}^{N}\right) \text {. }
$$

Then $u \equiv 0$.

Combining Corollary 1.3 and the Hardy-Littlewood-Sobolev inequality, we have a uniqueness result.

Corollary 1.4 (Uniqueness of Riesz potential). Let $s \in(0,1), 1<p<\frac{N}{2 s}$ and $f \in L^{p}\left(\mathbb{R}^{N}\right)$. Then there exists a unique $u \in L^{\frac{N p}{N-2 s p}}\left(\mathbb{R}^{N}\right)$ such that

$$
(-\Delta)^{s} u=f \quad \text { in } \mathcal{D}^{\prime}\left(\mathbb{R}^{N}\right),
$$

and $u$ is given by

where

$$
u(x)=\alpha_{N, s} \int_{\mathbb{R}^{N}} \frac{f(y)}{|x-y|^{N-2 s}} d y,
$$

$$
\alpha_{N, s}=\pi^{N / 2} 2^{2 s} \frac{\Gamma(s)}{\Gamma((N-2 s) / 2)} .
$$

The paper is organized as follows. In Section 2 we collect some basic facts concerning the fractional Laplacian $-(-\Delta)^{s}$ and $s$-harmonic functions. Finally, in Section 3 we prove the Cauchy-type estimate (1.1), the main result and its corollaries.

Note added in proof. We mention that after this paper was submitted, Liouvilletype results for a class of nonlocal operators were proved in [8] and [10] using Fourier transform.

\section{Preliminaries}

This section is devoted to recalling some basic notions about $s$-harmonic functions. We refer the reader to [7, Section 3]. Let $\mathcal{L}_{s}^{1}$ denote the space of all measurable functions $u: \mathbb{R}^{N} \rightarrow \mathbb{R}$ such that

$$
\int_{\mathbb{R}^{N}} \frac{|u(x)|}{1+|x|^{N+2 s}} d x<\infty .
$$

For functions $\varphi \in C^{2}\left(\mathbb{R}^{N}\right) \cap \mathcal{L}_{s}^{1}$, the fractional Laplacian $-(-\Delta)^{s}$ is defined by

$$
-(-\Delta)^{s} \varphi(x)=C_{N, s} \lim _{\varepsilon \rightarrow 0} \int_{|x-y|>\varepsilon} \frac{\varphi(y)-\varphi(x)}{|y-x|^{N+2 s}} d y \quad \text { for all } x \in \mathbb{R}^{N},
$$

where $C_{N, s}=s(1-s) \pi^{-N / 2} 4^{s} \frac{\Gamma\left(\frac{N}{2}+s\right)}{\Gamma(2-s)}$. 
For $u \in \mathcal{L}_{s}^{1}$, the expression $(-\Delta)^{s} u$ defines a distribution on every open set $\Omega \subset \mathbb{R}^{N}$ by

$$
\left\langle(-\Delta)^{s} u, \varphi\right\rangle=\int_{\mathbb{R}^{N}} u(x)(-\Delta)^{s} \varphi(x) d x \quad \text { for every } \varphi \in C_{c}^{\infty}(\Omega) .
$$

In the case where $(-\Delta)^{s} u=0$ in $\mathcal{D}^{\prime}(\Omega)$, we will say that $u$ is s-harmonic in $\Omega$. We note that affine functions $u$ belong to $\mathcal{L}_{s}^{1}$ if $s>1 / 2$ and constant functions $u$ belong to $\mathcal{L}_{s}^{1}$ if $s \in(0,1 / 2]$. Moreover, by using (2.1), in both cases, we can see that $(-\Delta)^{s} u(x)=0$ for every $x \in \mathbb{R}^{N}$. Furthermore, thanks to [7, Lemma 3.3], we have $(-\Delta)^{s} u=0$ in $\mathcal{D}^{\prime}\left(\mathbb{R}^{N}\right)$.

The fractional Laplacian has an explicit Poisson kernel with respect to the ball $B(x, r)$ (see [4]). It is given by

$$
P_{r}(x, y)=\left\{\begin{array}{l}
\beta_{N, s} \frac{\left(r^{2}-|x|^{2}\right)^{s}}{\left(|y|^{2}-r^{2}\right)^{s}}|y-x|^{-N} \text { for }|x|<r,|y|>r, \\
0 \quad \text { otherwise, }
\end{array}\right.
$$

where $\beta_{N, s}=\Gamma(N / 2) \pi^{-N / 2-1} \sin (s \pi)$. Therefore (see also [5]), if $u$ is $s$-harmonic in $\Omega$, then for every ball $B(a, r) \Subset \Omega$ we have

$$
u(x)=\int_{\mathbb{R}^{N}} P_{r}(x-a, y-a) u(y) d y \quad \text { for all } x \in B(a, r) .
$$

We now consider the regularization of $P_{r}$ as in [7]. To this end, we pick a function $\phi \in C_{c}^{\infty}(1,4)$ such that $\int_{\mathbb{R}} \phi(r) d r=1$ and define $\Psi: \mathbb{R}^{N} \rightarrow \mathbb{R}$ by

$$
\Psi(y)=\int_{1}^{4} P_{r}(0, y) \phi(r) d r=\beta_{N, s}|y|^{-N} \int_{\min (1,|y|)}^{\min (4,|y|)} r^{2 s}\left(|y|^{2}-r^{2}\right)^{-s} \phi(r) d r .
$$

Observe that, if $|y| \leq 1$, then $(0,|y|) \cap(1,4)=\emptyset$ and thus

$$
\Psi(y)=0 \quad \text { for every } y \in B(0,1) .
$$

Furthermore, as shown e.g. in [7, Lemma 3.11], we have $\Psi \in C^{\infty}\left(\mathbb{R}^{N}\right)$. Moreover, for every $\gamma \in \mathbb{N}^{N}$ there holds

$$
\left|D^{\gamma} \Psi(y)\right| \leq C|y|^{-N-2 s-|\gamma|} \quad \text { for every } y \in \mathbb{R}^{N} \backslash\{0\},
$$

where $C=C(N, \gamma, s)$ denotes, here and in the following, a positive constant depending only on $N, \gamma$ and $s$.

We define $\Psi_{r_{0}}(y)=r_{0}^{-N} \Psi\left(y / r_{0}\right)$, for $y \in \mathbb{R}^{N}$ and $r_{0}>0$. Then, for any $s$ harmonic function $u$ in an open set $\Omega \subset \mathbb{R}^{N}$, we have

$$
u(x)=u \star \Psi_{r_{0}}(x) \quad \text { for all almost every } x \in \Omega_{4 r_{0}},
$$

where $\Omega_{4 r_{0}}=\left\{x \in \Omega: \operatorname{dist}\left(x, \mathbb{R}^{N} \backslash \Omega\right)>4 r_{0}\right\}$; see [11, Lemma 2.6] or [7, Page 65]. We will therefore assume, in the sequel, that $s$-harmonic functions in some open set are smooth in that set.

\section{Proof of the main Result and its Consequences}

The following result (from which we will derive our main result) can be seen as a nonlocal version of the Cauchy estimate for bounded harmonic functions; see e.g. [3. Chapter 2]. 
Lemma 3.1. For every $\gamma \in \mathbb{N}^{N}$, there exists a constant $C>0$ only depending on $N, \gamma$ and $s$ such that for every function $u$ which is s-harmonic in $B(0, R)$,

$$
\left|D^{\gamma} u(0)\right| \leq C R^{2 s-|\gamma|} \int_{|y| \geq R / 4}|u(y)||y|^{-N-2 s} d y .
$$

Proof. Let $u$ be an $s$-harmonic function in $B(0, R)$ and $r_{0} \in(0, R / 4)$. Then by (2.5) we have

$$
u(x)=u \star \Psi_{r_{0}}(x) \quad \text { for all } x \in B\left(0, R-4 r_{0}\right) .
$$

By (2.3), (2.4) and the dominated convergence theorem, we deduce that

$$
D^{\gamma} u(0)=u \star D^{\gamma} \Psi_{r_{0}}(0) \quad \text { for all } \gamma \in \mathbb{N}^{N} .
$$

Using once more (2.3) and (2.4), we get

$$
\left|D^{\gamma} u(0)\right|=\left|\int_{|y| \geq r_{0}} u(y) D^{\gamma} \Psi_{r_{0}}(-y) d y\right| \leq C r_{0}^{2 s} \int_{|y| \geq r_{0}}|u(y)||y|^{-N-2 s-|\gamma|} d y .
$$

It follows that

$$
\left|D^{\gamma} u(0)\right| \leq C r_{0}^{2 s-|\gamma|} \int_{|y| \geq r_{0}}|u(y)||y|^{-N-2 s} d y .
$$

Letting $r_{0} \rightarrow R / 4$, we get the desired estimate.

As a consequence of Lemma 3.1, we have the following result.

Corollary 3.2. Let $\Omega$ be a nonempty open set of $\mathbb{R}^{N}$ such that $\Omega \neq \mathbb{R}^{N}$. Then for every $\gamma \in \mathbb{N}^{N}$, there exists a constant $C>0$ only depending on $N, \gamma$ and s such that for every function $u$ which is s-harmonic in $\Omega$,

$$
\left|D^{\gamma} u(x)\right| \leq C \delta_{\Omega}^{2 s-|\gamma|}(x) \int_{|y| \geq \frac{\delta_{\Omega}(x)}{4}}|u(y)||y|^{-N-2 s} d y \quad \text { for all } x \in \Omega,
$$

where $\delta_{\Omega}(x)=\operatorname{dist}\left(x, \mathbb{R}^{N} \backslash \Omega\right)$.

Proof. By assumption, $\delta_{\Omega}(x)<\infty$ for every $x \in \Omega$. Since $B\left(x, \delta_{\Omega}(x)\right) \subset \Omega$, applying Lemma 3.1 to the function $y \mapsto u(y+x)$ and $R=\delta_{\Omega}(x)$, we get the desired result.

Proof of Theorem 1.1. Let $x \in \mathbb{R}^{N}$ and $r>0$. We apply Corollary 3.2 with $\Omega=$ $B(x, r)$ and $|\gamma| \geq 2 s$. Then, letting $r \rightarrow \infty$, we get $\left|D^{\gamma} u(x)\right|=0$ for every $|\gamma| \geq 2 s$ and $x \in \mathbb{R}^{N}$. The proof of the theorem is thus completed.

Remark 3.3. It is well known that there are smooth functions $u$ - hence in $L_{\text {loc }}^{1}\left(\mathbb{R}^{N}\right)$ — satisfying $\Delta u=0$ in $\mathcal{D}^{\prime}\left(\mathbb{R}^{N}\right)$ for $N \geq 2$ which are not polynomials. Therefore a natural question arises: does there exist a larger space of distributions, strictly containing $\mathcal{L}_{s}^{1}$, where the fractional Laplacian is appropriately defined and where there are nontrivial entire $s$-harmonic functions which are not affine?

Proof of Theorem 1.2. The proof will be done by induction. Suppose $\ell$ is the degree of $P$. Assume that $\ell=0$ so that $P$ is a constant. Let $h \in \mathbb{R}^{N}$ and $u_{h}(x)=$ $u(x+h)-u(x)$. It is clear that $u_{h} \in \mathcal{L}_{s}^{1}$. In addition $(-\Delta)^{s} u_{h}=0$. It follows from Theorem 1.1 that $\partial_{i, j} u_{h}(0)=0$ and therefore $\partial_{i, j} u(h)=\partial_{i, j} u(0)$ for every $h \in \mathbb{R}^{N}$. This implies that $u$ is a second order polynomial and since it belongs to $\mathcal{L}_{s}^{1}$, it is affine. 
Now assume that the result holds true for a polynomial of degree up to $\ell \geq 0$ and suppose that $(-\Delta)^{s} u=P_{\ell+1}$, a polynomial of degree $\ell+1$. Then, for $h \in \mathbb{R}^{N}$, using the binomial formula we can see that $(-\Delta)^{s} u_{h}=P_{\ell, h}$, where $P_{\ell, h}$ is a polynomial of degree $\ell$. It follows from our assumption that $u_{h}$ is affine for any $h \in \mathbb{R}^{N}$. This again implies that $u$ is a second order polynomial and thus an affine function, since it belongs to $\mathcal{L}_{s}^{1}$.

Proof of Corollary 1.3. We just note that $L^{q}\left(\mathbb{R}^{N}\right) \subset \mathcal{L}_{s}^{1}$ for every $q \in[1, \infty]$ by Hölder's inequality.

Proof of Corollary 1.4. We define the function $\widetilde{u}(x)=\alpha_{N, s} \int_{\mathbb{R}^{N}} \frac{f(y)}{|x-y|^{N-2 s}} d y$. Let $f_{n} \in C_{c}^{\infty}\left(\mathbb{R}^{N}\right)$ be such that $f_{n} \rightarrow f$ in $L^{p}\left(\mathbb{R}^{N}\right)$. Define $u_{n}(x)=\alpha_{N, s} \int_{\mathbb{R}^{N}} \frac{f_{n}(y)}{|x-y|^{N-2 s}} d y$. By the Hardy-Littlewood-Sobolev inequality (see [12, Theorem 4.3]), we have $u_{n} \rightarrow$ $\widetilde{u}$ in $L^{\frac{N p}{N-2 s p}}\left(\mathbb{R}^{N}\right)$. In particular, $u_{n} \rightarrow \widetilde{u}$ in $\mathcal{L}_{s}^{1}$ by Hölder's inequality. Thanks to [6. Lemma 5.3], we have $(-\Delta)^{s} u_{n}=f_{n}$ in $\mathcal{D}^{\prime}\left(\mathbb{R}^{N}\right)$. Passing to the limit as $n \rightarrow \infty$, we deduce that $(-\Delta)^{s} \widetilde{u}=f$ in $\mathcal{D}^{\prime}\left(\mathbb{R}^{N}\right)$. Finally, if $u \in L^{\frac{N p}{N-2 s p}}\left(\mathbb{R}^{N}\right)$ is an arbitrary solution to $(-\Delta)^{s} u=f$ in $\mathcal{D}^{\prime}\left(\mathbb{R}^{N}\right)$, then $(-\Delta)^{s}(u-\widetilde{u})=0$ in $\mathcal{D}^{\prime}\left(\mathbb{R}^{N}\right)$. We thus conclude, from Corollary 1.3 that $u=\widetilde{u}$.

\section{REFERENCES}

[1] David Applebaum, Lévy processes - from probability to finance and quantum groups, Notices Amer. Math. Soc. 51 (2004), no. 11, 1336-1347. MR2105239 (2005j:60096)

[2] N. Abatangelo, Large s-harmonic functions and boundary blow-up solutions for the fractional laplacian, Discrete Contin. Dyn. Syst. A, 35; (2015), no. 12, 5555-5607.

[3] Sheldon Axler, Paul Bourdon, and Wade Ramey, Harmonic function theory, 2nd ed., Graduate Texts in Mathematics, vol. 137, Springer-Verlag, New York, 2001. MR.1805196 (2001j:31001)

[4] R. M. Blumenthal, R. K. Getoor, and D. B. Ray, On the distribution of first hits for the symmetric stable processes., Trans. Amer. Math. Soc. 99 (1961), 540-554. MR0126885 (23 \#A4179)

[5] K. Bogdan, T. Kulczycki, and Adam Nowak, Gradient estimates for harmonic and qharmonic functions of symmetric stable processes, Illinois J. Math. 46 (2002), no. 2, 541-556. MR 1936936 (2004b:60188)

[6] Krzysztof Bogdan and Tomasz Byczkowski, Potential theory of Schrödinger operator based on fractional Laplacian, Probab. Math. Statist. 20 (2000), no. 2, Acta Univ. Wratislav. No. 2256, 293-335. MR1825645 (2002a:31002)

[7] Krzysztof Bogdan and Tomasz Byczkowski, Potential theory for the $\alpha$-stable Schrödinger operator on bounded Lipschitz domains, Studia Math. 133 (1999), no. 1, 53-92. MR.1671973 (99m:31010)

[8] Wenxiong Chen, Lorenzo D'Ambrosio, and Yan Li, Some Liouville theorems for the fractional Laplacian, Nonlinear Anal. 121 (2015), 370-381, DOI 10.1016/j.na.2014.11.003. MR3348929

[9] Fausto Ferrari and Igor E. Verbitsky, Radial fractional Laplace operators and Hessian inequalities, J. Differential Equations 253 (2012), no. 1, 244-272, DOI 10.1016/j.jde.2012.03.024. MR2917408

[10] M. M. Fall and T. Weth, Liouville theorems for a general class of nonlocal operators, http://arxiv.org/abs/1504.00419.

[11] M. M. Fall and T. Weth, Monotonicity and nonexistence results for some fractional elliptic problems in the half space, http://arxiv.org/abs/1309.7230 (To appear in Com. Contemp. Math. DOI: 10.1142/S0219199715500121)

[12] Elliott H. Lieb and Michael Loss, Analysis, 2nd ed., Graduate Studies in Mathematics, vol. 14, American Mathematical Society, Providence, RI, 2001. MR1817225(2001i:00001)

[13] Edward Nelson, A proof of Liouville's theorem, Proc. Amer. Math. Soc. 12 (1961), 995. MR.0259149(41 \#3791) 
[14] X. Ros-Oton and J. Serra, Regularity theory for general stable operators, http://arxiv.org/ abs/1412.3892.

[15] R. Zhuo, W. Chen, X. Cui and Z. Yuan, A Liouville theorem for the fractional Laplacian, http://arxiv.org/pdf/1401.7402.

African Institute for Mathematical Sciences in Senegal, KM 2, Route de Joal, B.P. 14 18. Mbour, SÉnÉGAL

E-mail address: mouhamed.m.fall@aims-senegal.org

E-mail address: mouhamed.m.fall@gmail.com 